\title{
Hopf bifurcation control for the main drive delay system of rolling mill
}

\author{
Jinbin Wang ${ }^{1,2}$, Lifeng Ma ${ }^{1,2^{*}}$ and Yong Wang ${ }^{3}$
}

\section{"Correspondence:}

malifengasd@163.com

${ }^{1}$ College of Mechanical Engineering,

Taiyuan University of Science and

Technology, Taiyuan, China

${ }^{2}$ Engineering Research Center

Heavy Machinery Ministry of

Education, Taiyuan University of

Science and Technology, Taiyuan,

China

Full list of author information is

available at the end of the article

\section{Springer}

\begin{abstract}
In this work, the Hopf bifurcation of the main drive delay system of rolling mill is controlled and analyzed by designing a nonlinear controller. The time-delay is selected as a bifurcation parameter, and the following conclusions are obtained through analysis: (1) in the absence of state feedback control, the system will generate the Hopf bifurcation at the expense of its stability when the bifurcation parameter exceeds the threshold value; (2) in the state under feedback control, the occurrence of Hopf bifurcation is effectively delayed and the stable region of the system is also well extended. More importantly, we can change the properties of bifurcation periodic solutions by selecting the appropriate gain parameters. Some numerical simulations reveal that under the nonlinear feedback control, the vibration amplitude of the system can be effectively reduced.
\end{abstract}

Keywords: Main drive delay system of rolling mill; Hopf bifurcation; Bifurcation control; Nonlinear state feedback; Delay

\section{Introduction}

For decades, we have witnessed the development and improvement of nonlinear dynamics theory. The rolling mill vibration model [1-3] is a class of nonlinear dynamic systems that evolves from a simple linear model to a complex nonlinear dynamic model. According to the structure and load characteristics of the actual equipment, the nonlinear torsional vibration model is established with time delay [4], nonlinear stiffness [5, 6], and damping as nonlinear characteristic parameters to reveal more vibration mechanism and phenomena.

Many studies have shown that frictional vibrations encountered in actual production often cause bifurcations, which can seriously threaten the stable operation of the system. The Hopf bifurcation [7-11] is a common and important bifurcation phenomenon. The so-called Hopf bifurcation refers to the phenomenon that a closed orbit will occur in the vicinity equilibrium point when the stability of the singularity of the system is reversed, which can be used to explain many of the vibration problems in engineering.

In view of the system instability caused by Hopf bifurcation, some literature works [1216] have studied the various bifurcation control methods to modify the bifurcation characteristics, which can obtain some expected dynamical behaviors, for example, in order to postpone the onset of Hopf bifurcation [17], to change the properties of Hopf bifurcation [18], and to reduce the amplitude of vibration [19]. Xiao et al. [20] controlled unstable or

(c) The Author(s) 2020. This article is licensed under a Creative Commons Attribution 4.0 International License, which permits use sharing, adaptation, distribution and reproduction in any medium or format, as long as you give appropriate credit to the original author(s) and the source, provide a link to the Creative Commons licence, and indicate if changes were made. The images or other third party material in this article are included in the article's Creative Commons licence, unless indicated otherwise in a credit line to the material. If material is not included in the article's Creative Commons licence and your intended use is not permitted by statutory regulation or exceeds the permitted use, you will need to obtain permission directly from the copyright holder. To view a copy of this licence, visit http://creativecommons.org/licenses/by/4.0/. 
steady states and periodic orbits for a novel congestion control model by using the state feedback method. Xu [21] et al. used the polynomial function as a state feedback controller to realize the control of Hopf bifurcation for an internet congestion system.

In 2014, Zhang et al. established the main drive delay system of rolling mill [4]:

$$
\ddot{\theta}+\zeta \theta+\xi \dot{\theta}+\eta \dot{\theta}^{2}+\beta^{\prime} \dot{\theta}^{3}=p^{\prime} \theta(t-\tau)
$$

where $\zeta, \xi, \eta, \tau, c^{\prime}$, and $p^{\prime}$ are the real parameters. The specific meaning of parameters can be seen in [4].

Zhang et al. only gave the conditions for Hopf bifurcation to exist and some properties of bifurcating periodic solutions were not discussed. Furthermore, works seldom pay attention to the problem of Hopf bifurcation control for the main drive delay system of rolling mill. Our work will adopt the state feedback to control Hopf bifurcations. The main contributions of this paper are as follows:

(1) A nonlinear controller is established to control the Hopf bifurcation of the main drive delay system of rolling mill;

(2) The conditions for the existence of Hopf bifurcation in the main drive delay system of the rolling mill without control and with control are given, respectively;

(3) In the state under feedback control, the occurrence of Hopf bifurcation is effectively delayed and the nature of bifurcating periodic solutions can be changed by selecting proper gain parameters of the nonlinear part of the controller.

\section{Hopf bifurcation without control}

Firstly, let $x_{1}=\theta, x_{2}=\dot{\theta}, a_{1}=\xi, a_{2}=\eta, a_{3}=\beta^{\prime}, a_{4}=\zeta, a_{5}=p^{\prime}$, and system (1) becomes

$$
\left\{\begin{array}{l}
\dot{x_{1}}=x_{2}, \\
\dot{x_{2}}=-a_{1} x_{2}-a_{2} x_{2}^{2}-a_{3} x_{2}^{3}-a_{4} x_{1}+a_{5} x_{1}(t-\tau) .
\end{array}\right.
$$

The characteristic equation of system (2) at the zero equilibrium point $E_{0}=(0,0)^{T}$ is

$$
\lambda^{2}+a_{1} \lambda+a_{4}-a_{5} e^{-\lambda \tau}=0
$$

If $a_{1}>0, a_{4}>a_{5}, \tau=0$, then we can obtain that the equilibrium point $E_{0}$ is locally asymptotically stable.

If $\lambda=i \omega_{1}\left(\omega_{1}>0\right)$ is a solution of Eq. (3), then

$$
\begin{aligned}
& -\omega_{1}^{2}+a_{4}-a_{5} \cos \omega_{1} \tau=0 \\
& a_{1} \omega_{1}+a_{5} \sin \omega_{1} \tau=0,
\end{aligned}
$$

which can lead to

$$
\omega_{1}^{4}+\left(a_{1}-2 a_{4}\right) \omega_{1}^{2}+\left(a_{4}^{2}-a_{5}^{2}\right)=0
$$

On substituting, let $\omega_{1}^{2}=z, P_{1}=a_{1}^{2}-2 a_{4}, P_{2}=a_{4}^{2}-a_{5}^{2}$, Eq. (5) can be rewritten as

$$
z^{2}+P_{1} z+P_{2}=0
$$

and we define $h(z)=z^{2}+P_{1} z+P_{2}, z>0$. 
If $P_{2}<0$, then Eq. (6) has only a positive root $z_{1,2}=\frac{-P_{1}+\sqrt{\Delta}}{2}$, where $\Delta=P_{1}^{2}-4 P_{2}$. Thus, if $P_{2}<0$ holds, $\pm i \omega_{1}^{c}$ is a pair of purely imaginary roots of Eq. (3) with $\tau_{k 1}(k=0,1, \ldots)$, where $\tau_{k 1}=\frac{1}{\omega_{1}^{c}}(\theta+2 k \pi), \theta=\arcsin \left(\frac{a_{1} \omega_{1}^{c}}{-a_{5}}\right), \omega_{1}^{c}=\sqrt{z_{1,2}}=\sqrt{\frac{-P_{1}+\sqrt{\Delta}}{2}}$. Suppose that $\lambda(\tau)=\alpha(\tau)+i \omega(\tau)$ is the root of (3) satisfying $\alpha\left(\tau_{k 1}\right)=0, \omega\left(\tau_{k 1}\right)=\omega_{1}^{c}(k=0,1,2, \ldots)$.

Theorem 1 If $P_{1}<0, z \in\left(-\frac{P_{1}}{2},+\infty\right)$ holds, then $\operatorname{sign}\left(\left.\frac{d \operatorname{Re}(\tau)}{d \tau}\right|_{\tau=\tau_{k 1}}\right)=\operatorname{sign}\left(h^{\prime}(z)\right)>0$; if $P_{1}<$ $0, z \in\left(0,-\frac{P_{1}}{2}\right)$ holds, then $\operatorname{sign}\left(\left.\frac{d \operatorname{Re} \lambda(\tau)}{d \tau}\right|_{\tau=\tau_{k 1}}\right)=\operatorname{sign}\left(h^{\prime}(z)\right)<0$.

Proof Differentiating Eq. (3) with respect to $\tau$, we have

$$
\begin{aligned}
\frac{d \lambda}{d \tau} & =\frac{-a_{5} \lambda e^{-\lambda \tau}}{a_{1}+2 \lambda+a_{5} \tau e^{-\lambda \tau}} \\
& =\frac{-\lambda\left(\lambda^{2}+a_{1} \lambda+a_{4}\right)}{a_{1}+2 \lambda+a_{5} \tau e^{-\lambda \tau}}
\end{aligned}
$$

and

$$
\frac{d \operatorname{Re}\left(\lambda\left(\tau_{k}\right)\right)}{d \tau}=\frac{\omega^{2}}{\Lambda}\left[a_{1}^{2}-2 a_{4}+2 \omega_{1}^{2}\right]=\frac{\omega^{2}}{\Lambda}\left[h^{\prime}(z)\right] .
$$

Then

$$
\operatorname{sign}\left(\left.\frac{d \operatorname{Re} \lambda(\tau)}{d \tau}\right|_{\tau=\tau_{k 1}}\right)=\operatorname{sign}\left(h^{\prime}(z)\right)
$$

where $\Lambda=\left(a_{1}+a_{5} \tau \cos \left(\omega_{1} \tau_{k 1}\right)\right)^{2}+\left(2 \omega-a_{5} \tau \sin \left(\omega_{1} \tau_{k 1}\right)\right)^{2}$. By analyzing, we have the following results: (1) if $P_{1}<0, z \in\left(0,-\frac{P_{1}}{2}\right)$ holds, then $h^{\prime}(z)=2 z+P_{1}<0, z>0$; (2) if $P_{1}<0, z \in$ $\left(-\frac{P_{1}}{2},+\infty\right)$ holds, then $h^{\prime}(z)=2 z+P_{1}>0, z>0$. It completes the proof.

According to the above analysis, we have the following results.

Theorem 2 For system (2), suppose that $P_{2}<0$ holds.

(i) If $P_{1}<0, z \in\left(-\frac{P_{1}}{2},+\infty\right)$ holds, $E_{0}$ is locally asymptotically stable whenever $\tau \in\left[0, \tau_{01}\right)$ and $E_{0}$ is unstable whenever $\tau \in\left(\tau_{01},+\infty\right)$. Moreover, it generates a Hopf bifurcation at $E_{0}$ when $\tau=\tau_{k 1}$.

(ii) If $P_{1}<0, z \in\left(0,-\frac{P_{1}}{2}\right)$ holds, $E_{0}$ is locally asymptotically stable whenever $\tau \in\left[0, \tau_{01}\right) \cup\left(\bigcup_{i=1}^{\infty}\right)\left(\tau_{i, 1}, \tau_{i+1,1}\right)$. Furthermore, it generates a Hopf bifurcation at $E_{0}$ when $\tau=\tau_{k 1}$.

\section{Hopf bifurcation under control}

In this section, we design a nonlinear controller to control the Hopf bifurcation in the main drive delay system of rolling mill. The nonlinear state feedback controller is as follows:

$$
u=-b_{1} x_{1}-b_{2} x_{1}^{2}-b_{3} x_{1}^{3},
$$

where $b_{1}, b_{2}$, and $b_{3}$ are positive feedback parameters. The rolling mill main drive system Eq. (2) with state feedback controller can be rewritten as

$$
\left\{\begin{array}{l}
\dot{x_{1}}=x_{2}-b_{1} x_{1}-b_{2} x_{1}^{2}-b_{3} x_{1}^{3}, \\
\dot{x_{2}}=-a_{1} x_{2}-a_{2} x_{2}^{2}-a_{3} x_{2}^{3}-a_{4} x_{1}+a_{5} x_{1}(t-\tau) .
\end{array}\right.
$$


By calculating, system (8) has the characteristic equation

$$
\lambda^{2}+\left(a_{1}+b_{1}\right) \lambda+a_{4}+a_{1} b_{1}-a_{5} e^{-\lambda \tau}=0
$$

Let $\lambda=i \omega_{2}\left(\omega_{2}>0\right)$ be a root of Eq. (9), then

$$
\begin{aligned}
& -\omega_{2}^{2}+a_{4}+a_{1} b_{1}-a_{5} \cos \omega_{2} \tau=0, \\
& \left(a_{1}+b_{1}\right) \omega_{2}+a_{5} \sin \omega_{2} \tau=0
\end{aligned}
$$

and

$$
\omega_{2}^{4}+\left(a_{1}^{2}+b_{1}^{2}-2 a_{4}\right) \omega_{2}^{2}+\left(a_{4}+a_{1} b_{1}\right)^{2}-a_{5}^{2}=0 .
$$

Let $\omega_{2}^{2}=v, Q_{1}=a_{1}^{2}+b_{1}^{2}-2 a_{4}, Q_{2}=\left(a_{4}+a_{1} b_{1}\right)^{2}-a_{5}^{2}$, then Eq. (11) can be rewritten as

$$
v^{2}+Q_{1} v+Q_{2}=0
$$

and we define $h(v)=v^{2}+Q_{1} v+Q_{2}, v>0$.

By analyzing, if $Q_{2}<0$, then Eq. (12) has only a positive root $v_{1,2}=\frac{-Q_{1}+\sqrt{\Delta}}{2}$, where $\Delta=$ $Q_{1}^{2}-4 Q_{2}$. Thus, if $Q_{2}<0$ holds, $\pm i \omega_{2}^{c}$ is a pair of purely imaginary roots of Eq. (9) with $\tau_{k 2}(k=0,1, \ldots)$, where $\tau_{k 2}=\frac{1}{\omega_{2}^{c}}(\theta+2 k \pi), \theta=\arcsin \left(\frac{\left(a_{1}+b_{1}\right) \omega_{2}^{c}}{-a_{5}}\right), \omega_{2}^{c}=\sqrt{\nu_{1,2}}=\sqrt{\frac{-Q_{1}+\sqrt{\Delta}}{2}}$. Let $\lambda(\tau)=\alpha(\tau)+i \omega(\tau)$ be the root of (3) satisfying $\alpha\left(\tau_{k 2}\right)=0, \omega\left(\tau_{k 2}\right)=\omega_{2}^{c}(k=0,1,2, \ldots)$.

Similar to the analysis in the second part, the following conclusions can be drawn.

Theorem 3 If $Q_{1}<0, v \in\left(-\frac{Q_{1}}{2},+\infty\right)$ holds, then $\operatorname{sign}\left(\left.\frac{d \operatorname{Re} \lambda(\tau)}{d \tau}\right|_{\tau=\tau_{k 2}}\right)=\operatorname{sign}\left(h^{\prime}(v)\right)>0$; if $Q_{1}<$ $0, v \in\left(0,-\frac{Q_{1}}{2}\right)$ holds, then $\operatorname{sign}\left(\left.\frac{d \operatorname{Re} \lambda(\tau)}{d \tau}\right|_{\tau=\tau_{k 2}}\right)=\operatorname{sign}\left(h^{\prime}(v)\right)<0$.

Theorem 4 For system (8), suppose that $Q_{2}<0$ holds.

(i) If $Q_{1}<0, v \in\left(-\frac{Q_{1}}{2},+\infty\right)$ holds, $E_{0}$ is locally asymptotically stable whenever $\tau \in\left[0, \tau_{02}\right)$ and $E_{0}$ is unstable whenever $\tau \in\left(\tau_{02},+\infty\right)$. Moreover, it generates a Hopf bifurcation at $E_{0}$ when $\tau=\tau_{k 2}$.

(ii) If $Q_{1}<0, v \in\left(0,-\frac{Q_{1}}{2}\right)$ holds, $E_{0}$ is locally asymptotically stable whenever $\tau \in\left[0, \tau_{02}\right) \cup\left(\bigcup_{i=1}^{\infty}\right)\left(\tau_{i, 2}, \tau_{i+1,2}\right)$. Furthermore, it generates a Hopf bifurcation at $E_{0}$ when $\tau=\tau_{k 2}$.

In the following, we will explore the nature of the Hopf bifurcation for controlled system (8) by implementing the normal form (NF) and the center manifold reduction (CMR) [22].

Let $t \rightarrow \frac{t}{\tau}, \tau=\tau_{02}+\mu, \mu \in R$, then Eq. (8) can be written in a functional differential equation in $C=C\left([0,1], R^{2}\right)$ as follows:

$$
\dot{x(t)}=L_{\mu}(\phi)+F(\phi, \mu)
$$

where

$$
L_{\mu}(\phi)=\left(\tau_{02}+\mu\right)\left(\begin{array}{cc}
-b_{1} & 1 \\
-a_{4} & -a_{1}
\end{array}\right)\left(\begin{array}{l}
\phi_{1}(0) \\
\phi_{2}(0)
\end{array}\right)
$$




$$
\begin{gathered}
+\left(\tau_{02}+\mu\right)\left(\begin{array}{ll}
0 & 0 \\
a_{5} & 0
\end{array}\right)\left(\begin{array}{l}
\phi_{1}(-1) \\
\phi_{2}(-1)
\end{array}\right), \\
F(\mu, \phi)=\left(\tau_{02}+\mu\right)\left(\begin{array}{l}
-b_{2} \phi_{1}(0)^{2}-b_{3} \phi_{1}(0)^{3} \\
-a_{2} \phi_{2}(0)^{2}-a_{3} \phi_{2}(0)^{3}
\end{array}\right) .
\end{gathered}
$$

By Riesz representation theorem, let

$$
\eta(\theta, \mu)=\left(\tau_{02}+\mu\right)\left(\begin{array}{cc}
-b_{1} & 1 \\
-a_{4} & -a_{1}
\end{array}\right) \delta(\theta)-\left(\tau_{02}+\mu\right)\left(\begin{array}{cc}
0 & 0 \\
a_{5} & 0
\end{array}\right) \delta(\theta+1),
$$

where $\delta$ represents the Dirac delta function. Define

$$
A(\mu) \varphi= \begin{cases}\frac{\mathrm{d} \varphi(\theta)}{\mathrm{d} \theta}, & \theta \in[-1,0), \\ \int_{-1}^{0} d \eta(\theta, \mu) \varphi(\theta), & \theta=0,\end{cases}
$$

and

$$
R(\mu) \varphi= \begin{cases}0, & \theta \in[-1,0), \\ F(\mu, \varphi), & \theta=0\end{cases}
$$

where $\varphi \in C^{1}\left([-1,0] R^{2}\right)$. For $\psi \in C^{1}\left([0,1], R^{2}\right)$, define the adjoint operator of $A(\mu)$ as follows:

$$
A^{*} \psi(s)= \begin{cases}\int_{-1}^{0} d \eta(t, 0) \psi(-t), & s=0, \\ -\frac{d \psi(s)}{d s}, & s \in(0,1],\end{cases}
$$

and

$$
\langle\psi, \varphi\rangle=\bar{\psi}(0) \varphi(0)-\int_{-1}^{0} \int_{\xi=0}^{\theta} \bar{\psi}(\xi-\theta) d \eta(\theta) \varphi(\xi) d \xi
$$

$A(0)$ and $A^{*}$ are adjoint operators, and $A(0)$ has a pair of purely imaginary eigenvalues $\pm i \omega_{2}^{c} \tau_{02}$

Define $q(\theta)=\left(1, q_{2}(0)\right)^{T} e^{i \omega_{2}^{c} \tau_{02} \theta}, q^{*}(s)=D\left(1, q_{2}^{*}(0)\right)^{T} e^{i \omega_{2}^{c} \tau_{02} s}$. By calculating, we obtain $D=$ $\left(1+\overline{q_{2}(0)} q_{2}^{*}(0)-\tau_{02} q_{2}^{*}(0) a_{5} e^{i \omega_{0} \tau_{02}}\right)^{-1}, q_{2}(0)=i \omega_{2}^{c} \tau_{02}+b_{1}$ and $q_{2}^{*}(0)=\frac{1}{a_{1}-i \omega_{2}^{c} \tau_{02}}$.

On the center manifold $\Sigma_{0}$,

$$
w(z(t), \bar{z}(t), \theta)=w_{20}(\theta) \frac{z^{2}}{2}+w_{11}(\theta) z \bar{z}+w_{02}(\theta) \frac{\bar{z}^{2}}{2}+\cdots,
$$

we can get

$$
\begin{aligned}
\dot{z} & =i \omega \tau_{02} z+\left\langle q^{*}(\theta), F(W+2 \operatorname{Re} z(t) q(\theta))\right\rangle \\
& =i \omega \tau_{02} z+\bar{q}^{*}(0) F(W(z, \bar{z}, 0)+2 \operatorname{Re} z(t) q(\theta)) \\
& \doteq i \omega \tau_{02} z+\bar{q}_{0}^{*} F(z, \bar{z}),
\end{aligned}
$$


and this equation is rewritten as

$$
\dot{z}(t)=i \omega \tau_{02} z(t)+g(z, \bar{z})
$$

where

$$
\begin{aligned}
g(z, \bar{z}) & =\bar{q}_{0}^{*} F(W(z, \bar{z}, 0)+2 \operatorname{Re} z(t) q(\theta)) \\
& =g_{20} \frac{z^{2}}{2}+g_{11} z \bar{z}+g_{02} \frac{\bar{z}^{2}}{2}+g_{21} \frac{z^{2} \bar{z}}{2}+\cdots
\end{aligned}
$$

The following coefficients are obtained by using a computation similar to that of $[12,13]$, which are used for determining the important qualities

$$
\begin{aligned}
g_{20}= & -2 \bar{D} e^{2 i \tau_{02} \omega_{2}^{c} \theta}\left[a_{2} q_{2}^{2}(0) \overline{q_{2}^{*}(0)}+b_{2}\right] \\
g_{11}= & -\bar{D}\left[q_{2}^{*}(0) a_{2} q_{2}(0) \overline{q_{2}(0)}+b_{2}\right] \\
g_{20}= & -2 \bar{D} e^{-2 i \tau_{02} \omega_{2}^{c} \theta}\left[a_{2} \overline{q_{2}^{2}(0)} \overline{q_{2}^{*}(0)}+b_{2}\right] ; \\
g_{21}= & -2 \bar{D}\left[b_{2}\left(e^{\left.i \tau_{02} \omega_{2}^{c} \theta\right)}+e^{\left.-i \tau_{02} \omega_{2}^{c} \theta\right)} w_{20}^{1}\right)+a_{2} \overline{q_{2}^{*}(0)}\left(q_{2}(0) w_{11}^{2}(\theta) e^{\left.i \tau_{02} \omega_{2}^{c} \theta\right)}\right.\right. \\
& \left.\left.+\overline{q_{2}(0)} w_{20}^{2}(\theta) e^{-i \tau_{02} \omega_{2}^{c} \theta}\right)\right]
\end{aligned}
$$

where $w_{20}$ and $w_{11}$ satisfy

$$
\begin{aligned}
& w_{20}(\theta)=\frac{g_{20}}{i \omega_{2}^{c} \tau_{02}} q(0) e^{i \tau_{02} \omega_{2}^{c} \theta}-\frac{\bar{g}_{02}}{3 i \omega_{2}^{c} \tau_{02}} \bar{q}(0) e^{-i \tau_{02} \omega_{2}^{c} \theta}+E_{1} e^{2 i \omega \tau_{02} \theta}, \\
& w_{11}(\theta)=\frac{g_{11}}{i \omega_{2}^{c} \tau_{02}} q(0) e^{i \tau_{02} \omega_{2}^{c} \theta}-\frac{\bar{g}_{11}}{i \omega_{2}^{c} \tau_{02}} \bar{q}(0) e^{-i \tau_{02} \omega_{2}^{c} \theta}+E_{2} .
\end{aligned}
$$

Note that

$$
\begin{aligned}
& E_{1}=\left(2 i \omega_{2}^{c} I-\int_{-1}^{0} e^{i \tau_{02} \omega_{2}^{c} \theta} d \eta(\theta, 0)\right)^{-1} F_{z^{2}} \\
& E_{2}=-\left(\int_{-1}^{0} d \eta(\theta, 0)\right)^{-1} F_{z \bar{z}}
\end{aligned}
$$

we can get

$$
\begin{aligned}
& E_{1}=\left(\begin{array}{cc}
2 i \omega_{2}^{c} & -1 \\
a_{4}-a_{5} e^{-i \tau_{02} \omega_{2}^{c}} & 2 i \omega_{2}^{c}+a_{1}
\end{array}\right)^{-1}\left(\begin{array}{c}
2 b_{2} e^{2 i \tau_{02} \omega_{2}^{c} \theta} \\
2 a_{2} q_{2}(0)^{2} e^{2 i \tau_{02} \omega_{2}^{c} \theta}
\end{array}\right) \\
& E_{2}=\left(\begin{array}{cc}
0 & -1 \\
a_{4}-a_{5} & a_{1}
\end{array}\right)^{-1}\left(\begin{array}{c}
b_{2} \\
a_{2} q_{2}(0) \overline{q_{2}(0)}
\end{array}\right) .
\end{aligned}
$$

Therefore, by our previous analysis, we can obtain the following parameters that determine the nature of Hopf bifurcation:

$$
c_{1}(0)=\frac{i}{2 \omega_{2}^{c} \tau_{02}}\left(g_{20} g_{11}-2\left|g_{11}\right|^{2}-\frac{1}{3}\left|g_{02}\right|^{2}\right)+\frac{g_{21}}{2},
$$


Figure 1 The equilibrium of system (2) with $\tau=0.5$ $<\tau_{01}$ is stable
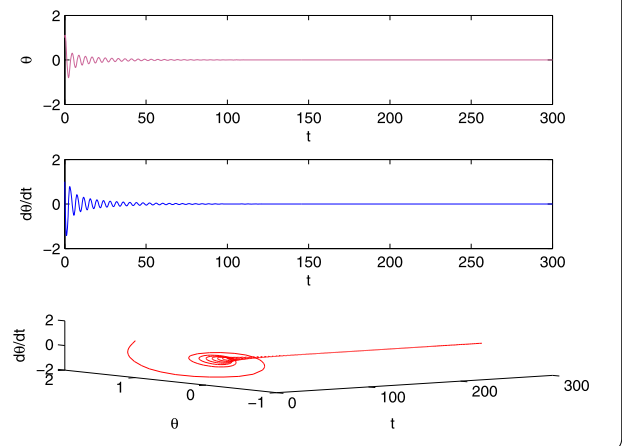

Figure 2 The bifurcation periodic solution of uncontrolled system (2) with $\tau=0.7>\tau_{01}$ is stable
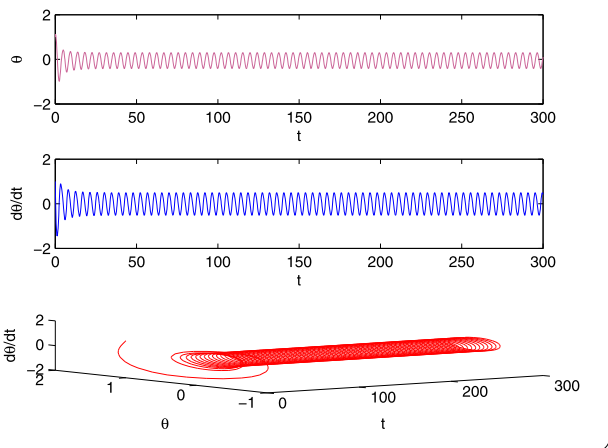

Figure 3 The waveform of system (2) without control at $\tau=2.3,2.7,3.1$, respectively
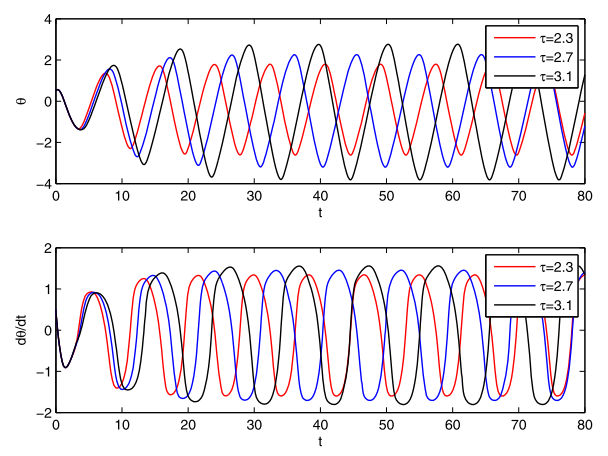

$$
\begin{aligned}
& \mu_{2}=-\frac{\operatorname{Re} c_{1}(0)}{\operatorname{Re} \lambda^{\prime}\left(\tau_{02}\right)}, \\
& \beta_{2}=2 \operatorname{Re} c_{1}(0), \\
& T_{2}=-\frac{\operatorname{Im} c_{1}(0)+\mu_{2} \operatorname{Im} \lambda^{\prime}\left(\tau_{02}\right)}{\omega_{2}^{c}} .
\end{aligned}
$$

Theorem 5 For system (8) with $\tau_{k 2}$, the following results hold:

(i) If $\mu_{2}>0\left(\mu_{2}<0\right)$, then the Hopf bifurcation is supercritical (subcritical).

(ii) If $\beta_{2}<0\left(\beta_{2}>0\right)$, then the bifurcating periodic solutions are stable (unstable).

(iii) If $T_{2}>0\left(T_{2}<0\right)$, then the period increases (decreases). 
Figure 4 Phase portrait of system (2) without control at $\tau=2.3,2.7,3.1$, respectively

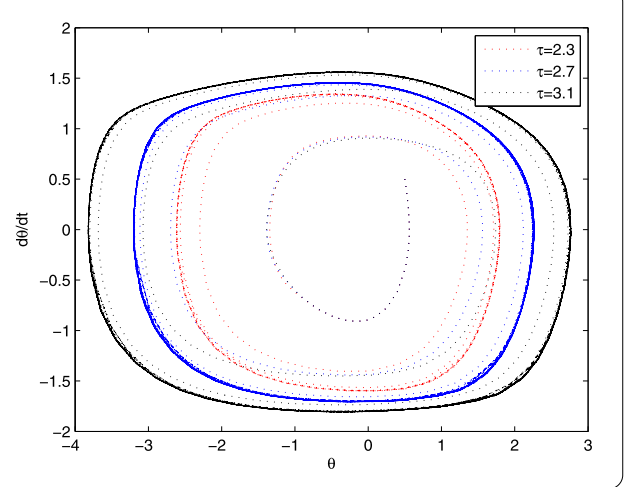

Figure 5 The equilibrium of system (8) with $\tau=1$ $<\tau_{02}$ is stable
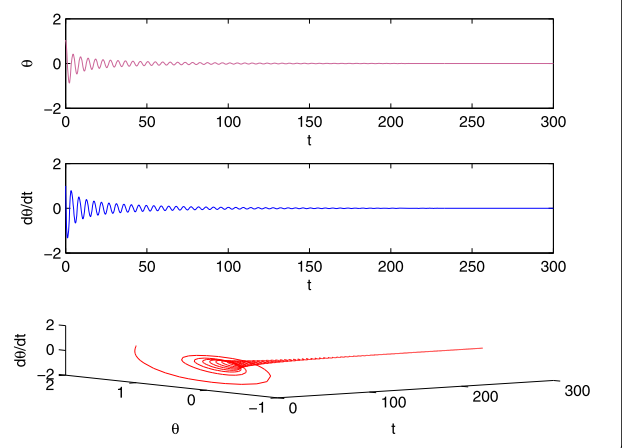

Figure 6 The bifurcation periodic solution of controlled system (8) with $\tau=1.2>\tau_{02}$ is stable
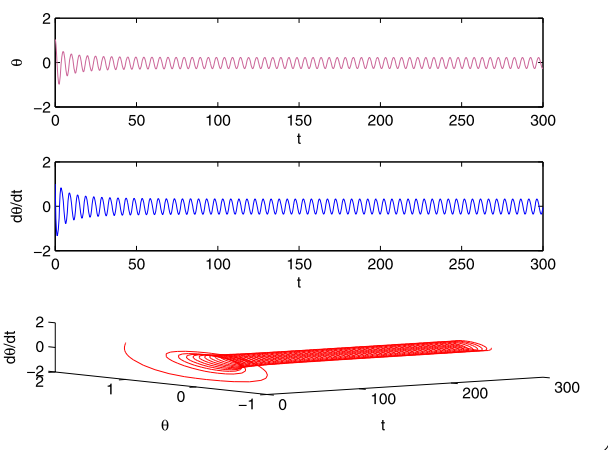

\section{Numerical simulation}

In this section, we implement some simulations to corroborate the obtained conclusions in the previous section.

Case 1: The system without control.

Fix parameters $a_{1}=1, a_{2}=a_{3}=a_{4}=1, a_{5}=-2$. From Theorem 2, we can get $P_{1}=-1<$ $0, P_{2}=-3<0, \omega_{1}^{c}=1.517, \tau_{01}=0.568$.

We choose $\tau=0.5<\tau_{01}=0.568, \tau \in\left[0, \tau_{01}\right)$ and note that the equilibrium of system (2) is stable, as shown in Fig. 1. As $\tau$ exceeds the critical value $\tau_{01}$, system (2) generates a Hopf bifurcation at the expense of its stability. By choosing $\tau=0.7>\tau_{01}=0.568$, Fig. 2 reveals that there exists a stable periodic solution. Figures 3,4 show that as the time delay increases, the vibration amplitude of the uncontrolled system increases.

Case 2: The system under control. 
Figure 7 Bifurcation diagram for $\theta$ versus $\tau$ of uncontrolled system (2) and controlled system (8), respectively
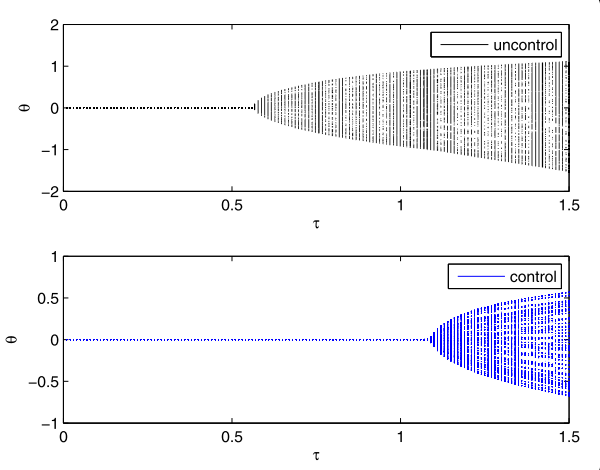

Figure 8 Bifurcation diagram for $\frac{d \theta}{d t}$ versus $\tau$ of uncontrolled system (2) and controlled system (8), respectively
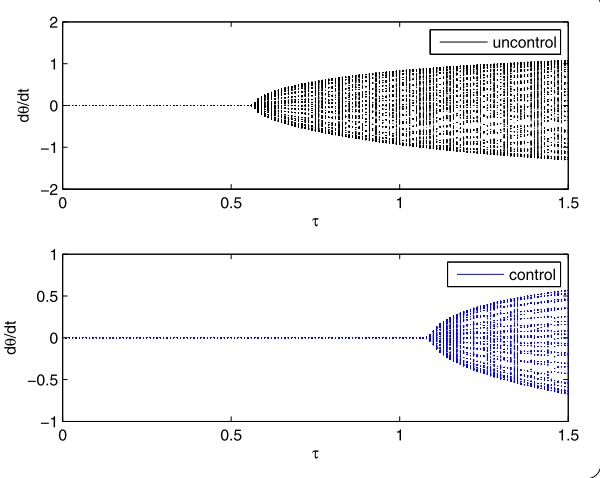

Figure 9 The stability domain of system (8) varies with the increase of the control parameter $b_{1}$

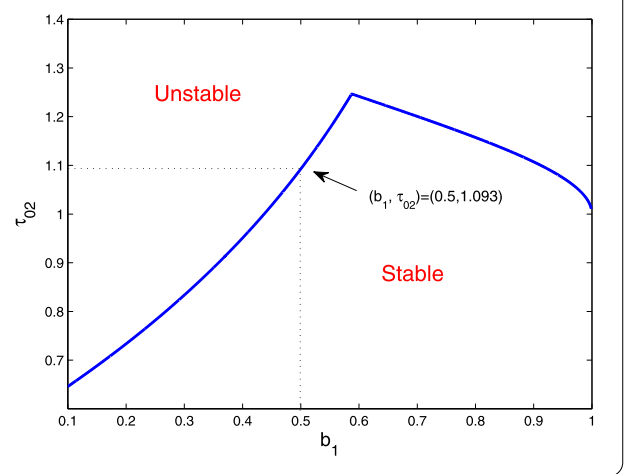

Fix parameters $a_{1}=1, a_{2}=a_{3}=a_{4}=1, a_{5}=-2, b_{1}=0.5, b_{2}=b_{3}=0.01$. From Theorem 4, we can get $Q_{1}=-0.75<0, Q_{2}=-1.75<0, \omega_{2}^{c}=1.323, \tau_{02}=1.093$.

We choose $\tau=1<\tau_{01}=1.093, \tau \in\left[0, \tau_{02}\right)$ and note that the equilibrium of system (8) is stable, as shown in Fig. 5. As $\tau$ exceeds the critical value $\tau_{02}$, system (8) generates a Hopf bifurcation at the expense of its stability. By choosing $\tau=1.2>\tau_{02}=1.093$, we can get $c_{1}(0)=-1.2736+5.3826 i, \mu=-0.7634, \beta_{2}=-2.5472$, as depicted in Fig. 6. Figures 7,8 show that the bifurcation point is delayed from 0.568 to 1.123 and demonstrate that the occurrence of the Hopf bifurcation can be effectively retarded.

Fix parameters $a_{1}=1, a_{2}=a_{3}=a_{4}=1, a_{5}=-2$. By choosing $b_{1}$ as a parameter, we can obtain $\tau_{02}=\frac{1}{\omega_{2}^{c}} \arcsin \left(\frac{\left(a_{1}+b_{1}\right) \omega_{2}^{c}}{-a_{5}}\right)$. From Theorem 4: if $\tau<\tau_{02}$ holds, $E_{0}$ is locally stable; if $\tau>\tau_{02}$ holds, $E_{0}$ is unstable whenever $\tau \in\left(\tau_{02},+\infty\right)$. Moreover, it suffers a Hopf bifurcation at $E_{0}$ when $\tau=\tau_{02}$. Stable domain and unstable domain of system (8) with the nonlinear 
Figure 10 Waveforms and phase diagrams of uncontrolled system (2) and controlled system (8) with $\tau=2.3$

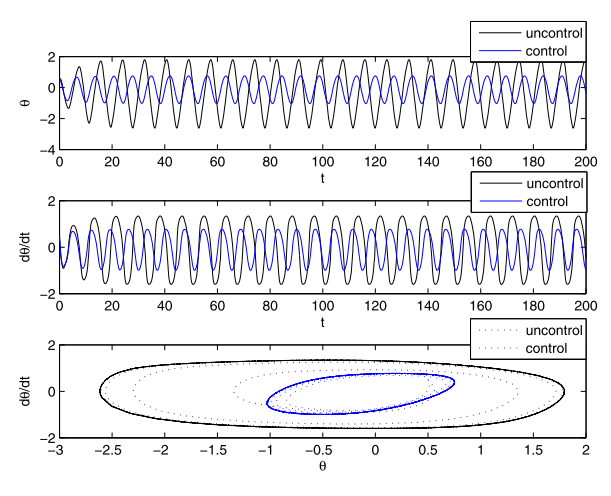

Figure 11 Waveforms and phase diagrams of uncontrolled system (2) and controlled system (8) with $\tau=2.7$

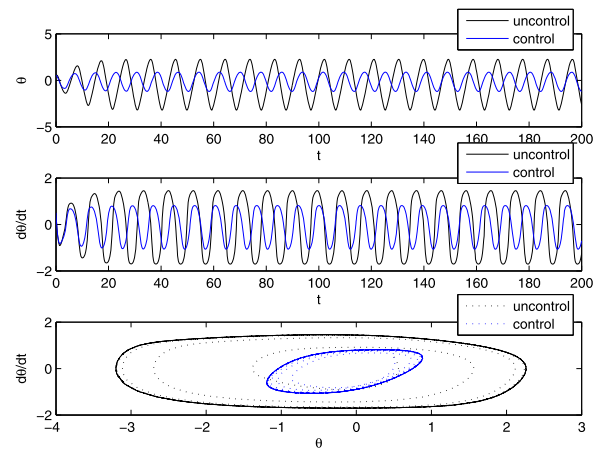

Figure 12 Waveforms and phase diagrams of uncontrolled system (2) and controlled system (8) with $\tau=3.1$

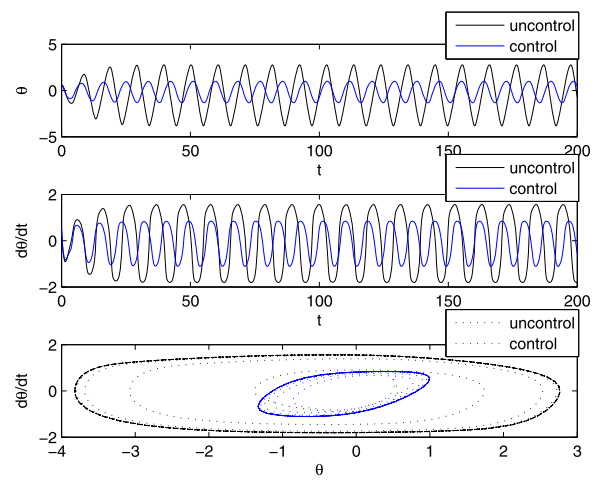

state feedback control are shown in Fig. 9. From Fig. 9, we note that the threshold value of the bifurcation increases with the increase of the control parameter $b_{1}$ and reaches the maximum value around $b_{1}=0.6$, then the threshold value decreases with the increase of $b_{1}$.

In Figs. 10, 11, 12, we show waveforms and phase diagrams of the uncontrolled system and controlled system (2) with the same time delay, respectively. The result indicates that the vibration amplitude of the system can be effectively reduced under nonlinear feedback control. Therefore, the proposed control strategy is feasible. 


\title{
5 Conclusion
}

In this paper, the main drive delay system of the rolling mill is considered and a nonlinear controller is designed to control the Hopf bifurcation in the system. We give the conditions for the Hopf bifurcation to exist in the main drive delay system of rolling mill without control and under control, respectively. In the state under feedback control, the occurrence of Hopf bifurcation is effectively delayed, and the nature of bifurcating periodic solutions can be changed by selecting proper gain parameters of the nonlinear part of the controller. Besides, the relation graph between the stability of system (8) and control parameter is given. Some numerical results validate the above analysis.

\author{
Acknowledgements \\ The authors very gratefully acknowledge the helpful comments of the editors and anonymous reviewers. \\ Funding \\ This work is supported by the National Natural Science Foundation of China (Nos. U1610253, 11701410), the Key Research \\ and Development Program of Shanxi Province (Nos. 201603D111004), and the Fund for Shanxi "1331 Project" Key \\ Subjects Construction.
}

Availability of data and materials

Data sharing not applicable to this article as no datasets were generated or analysed during the current study.

Competing interests

The authors declare that they have no competing interests.

Authors' contributions

All authors read and approved the final manuscript.

\section{Author details}

${ }^{1}$ College of Mechanical Engineering, Taiyuan University of Science and Technology, Taiyuan, China. ${ }^{2}$ Engineering Research Center Heavy Machinery Ministry of Education, Taiyuan University of Science and Technology, Taiyuan, China. ${ }^{3}$ Department of Information Science and Technology, Tianjin University of Finance and Economics, Tianjin, China.

\section{Publisher's Note}

Springer Nature remains neutral with regard to jurisdictional claims in published maps and institutional affiliations.

Received: 27 November 2019 Accepted: 28 April 2020 Published online: 13 May 2020

\section{References}

1. Lu, X., Sun, J., Li, G.T., Wang, Q.L., Zhang, D.H.: Dynamic analysis of vibration stability in tandem cold rolling mill. J. Mater. Process. Technol. 272, 47-57 (2019)

2. Wang, X.X., Yan, X.Q.: Dynamic model of the hot strip rolling mill vibration resulting from entry thickness deviation and its dynamic characteristics. Math. Probl. Eng. 2019, 1-11 (2019)

3. Zeng, L.Q., Zang, Y.: Gao, Z.Y.: Hopf bifurcation control for rolling mill multiple-mode-coupling vibration under nonlinear friction. J. Vib. Acoust. 139(6), 061015 (2019)

4. Zhang, R., Yang, P., Cui, C.: Hopf bifurcation for nonlinear delay system of rolling mill main drive. J. Vib. Meas. Diagn. 34(05), 909-914 (2014) (in Chinese)

5. Liu, S., Ai, H., Sun, B., et al.: Bifurcation and chaos of electromechanical coupling main drive system with strongly nonlinear characteristic in mill. Chaos Solitons Fractals 98, 101-108 (2017)

6. Gao, C.Y., Du, G.J., Feng, Y., et al.: Nonlinear vibration analysis of moving strip with inertial boundary condition. Math. Probl. Eng. 2015(8), 1-9 (2015)

7. Wei, J., Jiang, W.: Stability and bifurcation analysis in Van der Pol's oscillator with delayed feedback. J. Sound Vib. 283(3-5), 801-819 (2005)

8. Jiang, W., Wang, H.: Hopf-transcritical bifurcation in retarded functional differential equations. Nonlinear Anal. 73(11), 3626-3640 (2005)

9. Wang, Y., Wang, H., Jiang, W.: Stability switches and global Hopf bifurcation in a nutrient-plankton model. Nonlinear Dyn. 78(2), 981-994 (2014)

10. Li, Y., Jiang, W., Wang, H.: Double Hopf bifurcation and quasi-periodic attractors in delay-coupled limit cycle oscillators. J. Math. Anal. Appl. 387(2), 1114-1126 (2012)

11. Ding, Y., Jiang, W.: Double Hopf bifurcation and chaos in Liu system with delayed feedback. J. Appl. Anal. Comput. 1(3), 325-349 (2011)

12. Chen, Z., Yu, P.: Hopf bifurcation control for an Internet congestion model. Int. J. Bifurc. Chaos 15, 2643-2651 (2005)

13. Xiao, M., Cao, J.: Delayed feedback-based bifurcation control in an Internet congestion model. J. Math. Anal. Appl. 332, 1010-1027 (2007)

14. Ding, D., Zhu, J., Luo, X., Liu, Y.: Controlling Hopf bifurcation in fluid flow model of Internet congestion control system Int. J. Bifurc. Chaos 19, 1415-1424 (2009) 
15. Guo, S., Liao, X., Liu, Q., Li, C.: Necessary and sufficient conditions for Hopf bifurcation in exponential RED algorithm with communication delay. Nonlinear Anal., Real World Appl. 9, 1768-1793 (2008)

16. Liu, F., Wang, H., Guan, Z.: Hopf bifurcation control in the XCP for the Internet congestion control system. Nonlinear Anal., Real World Appl. 13, 1466-1479 (2012)

17. Abed, E.H., Wang, H.O., Chen, R.C.: Stabilization of period doubling bifurcations and implications for control of chaos. Physica D 70, 154-164 (1994)

18. Berns, D.W., Moiola, J.L., Chen, G.: Feedback control of limit cycle amplitude from a frequency domain approach. Automatica 34, 1567-1573 (1998)

19. Li, C., Chen, G., Liao, X., Yu, J.: Hopf bifurcation in an Internet congestion control model. Chaos Solitons Fractals 19 853-862 (2004)

20. Xiao, M., Jiang, G.P., Zhao, L.D.: State feedback control at Hopf bifurcation in an exponential RED algorithm model. Nonlinear Dyn. 76(2), 1469-1484 (2014)

21. Xu, W., Hayat, T., Cao, J., Xiao, M.: Hopf bifurcation control for a fluid flow model of Internet congestion control systems via state feedback. IMA J. Math. Control Inf. 33(1), 69-93 (2014)

22. Hassard, B.D., Kazarinoff, N.D., Wan, Y.H.: Theory and Applications of Hopf Bifurcation. Cambridge University Press, Cambridge (1981)

Submit your manuscript to a SpringerOpen ${ }^{\circ}$ journal and benefit from:

- Convenient online submission

- Rigorous peer review

- Open access: articles freely available online

- High visibility within the field

- Retaining the copyright to your article

Submit your next manuscript at $\boldsymbol{\triangleright}$ springeropen.com 